$10 / y^{1} / 8^{0}$

$D R .761$

MASTER

Extraction of the Michel Parameters from Muon Decay by a Maximum Likelihood Method

$\frac{\text { 민 }}{\frac{2}{0}}$

0

$\frac{2}{\frac{2}{0}}$ 


\title{
EXTRACTION OF THE MICHEL PARAMETERS FROM MUON DECAY BY A MAXIMUM LIKELIHOOD METHOD
}

by

\author{
R. J. McKee
}

\begin{abstract}
We have developed a maximum likelihood method for extracting the Michel parameters, which describe the decay spectrum of stopped pr'arized muons. The method was applied successfully in a Monte Carlo simulation of our apparatus. We also obtained complete error matrix for the set of Michel parameters. In an experiment of $10^{8}$ muon decays we can reduce the statistical errors by more than an order of magnitude over the currently accepted limits on the Michel parameters.
\end{abstract}

In our approved LAMPF experiment, we propose to measure the Michel spectrum of positrons from polarized muon decay and to extract the Michel parameters to an unprecedented accuracy. To achieve an order of magnitude improvement over previous results, ${ }^{1}$ we must measure $\sim 10^{8}$ muon decays in our time projection chamber (TPC). However, because so many data present handling and analysis problems, we have developed a more efficient method for extracting the four Michel parameters.

We compare the maximum likelihood method with the standard procedure, in which one separates (bins) the data in momentum and angle, then performs a least squares fit to the spectrum. The standard procedure, however, has inherent inaccuracies. Unless the bin size is considerably smaller than the resolution, an event can move around in its bin, which results in lo: $:$ information. To make the two-dimensional bins small enough for our experiment would require $10^{4}$ to $10^{5}$ bins, an awkwardly large number for performing a least squares fit.

With the inaximum likelihood method no information is lost because there is no binning. All the data are reduced to about 150 numbers, which are then used to extract the Michel parameters.

In this report we describe the method and present a numerical example based upon the apparatus we will use. We also display the complete Michel parameter error matrix, calculated in such a way that $\mathrm{N}$, the number of events, appears as a parameter. Thus, we can find the statistical errors on the Michel parameters for any amount of data simply by substituting $\mathrm{N}$ into our numerical results.

Neglecting the radiative corrections, we describe the Michel spectrum, which is a function of the emitted positron's energy and the angle the positron makes relative to the stationary muon's spin direction. The two variables $\mathrm{x}$ and $\mu$ in Eq. (1) refer to the energy relative to the maximum $\left(\mathrm{E}_{\mathrm{o}}=52.8 \mathrm{MeV}\right)$ and cosine of the angle. We write the Michel spectrum as 


$$
P_{0}(x, \mu)=\sum_{k=1}^{5} a_{k} F_{k}(x) \mu^{n_{k}},
$$

where $a_{k}$ and $n_{k}$ are as given below.

$$
\begin{array}{ccc}
\underline{\mathbf{k}} & \mathbf{a}_{\mathbf{k}} & \underline{\mathbf{n}_{\mathbf{k}}} \\
\hline ! & 1 & 0 \\
2 & \rho & 11 \\
: 3 & \eta & 0 \\
1 & 0 \xi & 1 \\
: & 0 \xi \delta & 1
\end{array}
$$

The four Michel parameters are $\rho, \eta, \xi$, and $\delta ; \mathrm{p}$ is the poiarization of the stopped muons in the TPC gas.

The $F_{k}(x)$ are simple functions of $x$. For our analysis, we simplified the $F_{x}(x)$ by ignoring the positron's mass, $m$, in most cases. The functions then become

$$
\begin{aligned}
& F_{1}(x)=3(1-x) x^{2}, \\
& F_{2}(x)=2\left(\frac{4}{3} x-1\right) x^{2}, \\
& F_{3}(x)=3 x_{0}(1-x) x, \\
& F_{4}(x)=(1-x) x^{2},
\end{aligned}
$$

and

$$
F_{5}(x)=2\left(\frac{4}{3} x-1\right) x^{2}
$$

where $\mathrm{x}_{\mathrm{o}}=\mathrm{mc}^{2} / \mathrm{E}_{\mathrm{o}}$.

For a given event the probability that the energy will fall between $x$ and $x+d x$, the angle between $\mu$ and $\mu+\mathrm{d} \mu$, is shown as

$$
P(x, \mu) d x d \mu \text {, }
$$

where the probability is related to $P_{0}(x, \mu)$ by

$$
P(x, \mu)=X^{-1} A(x, \mu) P_{0}(x, \mu)
$$

where $\mathrm{X}$ is the normalization factor and $\mathrm{A}(\mathrm{x}, \mu)$ is the TPC acceptance function. The acceptance function reflects not only the geometric acceptance but also any cuts one may wish to impose on the TPC track characteristics. The normalization factor $\mathrm{X}$ is a natural result of the condition

$$
\int_{0}^{1} d x \int_{-1}^{1} d \mu P(x, \mu)=1
$$


Hence,

$$
x=\sum_{k=1}^{5} a_{k} g_{k} .
$$

and

$$
g_{k}=\int_{0}^{1} F_{k}(x) d x \int_{-1}^{1} A(x, \mu)_{\mu}{ }^{n_{k}} d \mu .
$$

The five $g_{k}$ quantities are the acceptance factors.

To measure $\mathrm{N}$ muon decays, we use the set of measurements $\left\{\mathrm{x}_{\mathrm{i}}, \mu_{1}\right\}$. The probability of seeing that particular set is

$$
G\left(\rho, \eta, \xi, \delta ;\left(x_{i}, \mu_{i}\right\}\right)=\prod_{i=1}^{N} P\left(x_{i}, \mu_{j}\right) .
$$

The maximum likelihood method demands that we jiggle the four Michel parameters until $\mathrm{G}$ is maximized. Mathematically, we take he derivatives of $\ell n G$ with respect to the Michel parameters and set the results to zero to obtain

$$
\frac{x}{M} \sum_{i=1}^{N} \frac{F_{k}\left(x_{i}\right) \mu_{i}{ }^{n_{k}}}{P_{0}\left(x_{i}, \mu_{j}\right)}=g_{k}, k=2,3,4,5 .
$$

Although Eq. (9) can also be used for $\mathrm{k}=1$, it is not needed because it is a direct result of the other four parameters.

The four simultaneous equations expressed in Eq. (9) are very awkward. The four Michel parameters, buried in $\mathrm{X}$ and $\mathrm{P}_{\mathrm{o}}$, must be iterated in some way. Each iteration means a new calculation must be made of the sum in Eq. (9). The reading of the millions of $\left(x_{1}, \mu_{1}\right)$ pairs, which are on disk or tape, is very time-consuming. Therefore, if sums are required, it would be more efficient to read the data and do the sums only once. For this reason, we need a way of factoring out the Michel parameters from the sums.

We take $P_{0}(x, \mu)^{-1}$ and make a Taylor series expansion in the Michel parameters about the V-A values to obtain

$$
a_{k}=a_{k}^{\star}+\Delta a_{k},
$$

where $a_{k}^{*}$ is the $V$-A result. Equation (10) is also defined for $k=1$, but by definition $a_{i}^{*}=1$, and $\Delta a_{1}=0$. Therefore,

$$
P_{0}(x, \mu)=P_{0}^{*}(x, \mu)+\sum_{k=2}^{5} \Delta a_{k} F_{k}(x)_{\mu}{ }^{n_{k}},
$$


and

$$
x=x^{*}+\sum_{k=2}^{5} \Delta a_{k} g_{k}
$$

The expansion is then

$$
\begin{gathered}
P_{0}(x, \mu)^{-1}=\sum_{m=0}^{\infty}(-1)^{m} \sum_{k_{2}=0}^{m} \sum_{k_{3}=0}^{m-k_{2}} \sum_{k_{4}=0}^{m-k_{2}-k_{3}} \frac{m !}{k_{2} ! k_{3} ! k_{4} ! k_{5} !} \\
{\left[\prod_{\alpha=2}^{5}\left(\Delta a_{\alpha}\right)^{k_{\alpha}}\right] \frac{\prod_{\alpha=-}^{5}\left(F \alpha(x)_{\mu}{ }^{n_{\alpha}}\right)^{k_{\alpha}}}{P_{0}^{\star}(x, \mu)^{m+1}} .}
\end{gathered}
$$

where $k_{b}=m-\left(k_{2}+k_{a}+k_{4}\right)$. Equation (12) can be simplified by breaking it up and adding the missing factors from Eq. (9).

$$
\begin{aligned}
& R_{k_{2} k_{3} k_{4} k_{5}}^{k}=\frac{(-1)^{m} m !}{k_{2} ! k_{3} ! k_{4} ! k_{5} !} \frac{1}{N} \sum_{i=1}^{N} \frac{\prod_{\alpha=2}^{5}\left(F_{\alpha}\left(x_{1}\right) \mu_{1}{ }^{n_{\alpha}}\right)^{k_{\alpha}+\delta_{\alpha k}}}{P_{0}^{*}\left(x_{i}, \mu_{i}\right)^{m+1}}, \\
& k=2,3,4,5,
\end{aligned}
$$

and

$$
Q_{M k}=\sum_{m=0}^{M} \sum_{k_{2}=0}^{m} \sum_{k_{3}=0}^{m-k_{2}} \sum_{k_{4}=0}^{m-k_{2}-k_{3}} R_{k_{2} k_{3} k_{4} k_{5}}^{k} \prod_{\alpha=2}^{5}\left(\Delta a_{\alpha}\right)^{k_{\alpha}} .
$$

Equatio (9) now becomes

$$
\left(x^{*}+\sum_{\ell=2}^{5} g_{\ell} \Delta a_{\ell}\right) Q_{M k}=g_{k}, \quad k=2,3,4,5 .
$$

Note that we have introduced a cutnff $M$ in the infinite sum. It turns out that $M$ need not be very large. Of more importance, is"wever, is the isolation of the coveted sums in the $R$ factors; that is, we have reduced the millions of $\left(x_{1}, \mu_{1}\right)$ pairs to only a few $R$ factors.

To carry out the iteration in $a_{k}$, we define a modified $Q_{M k}$.

$$
Q_{M k}^{\prime}=\sum_{m=2}^{M} \sum_{k_{2}=0}^{m} \sum_{k_{3}=0}^{m-k_{2}} \sum_{k_{4}=0}^{m-k_{2}-k_{3}} R_{k_{2} k_{3} k_{4} k_{5}}^{k} \prod_{\alpha=2}^{5}\left(\Delta a_{\alpha}\right)^{k} .
$$


We also define

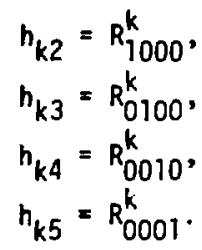

Then Eq. (15) can be written as

$$
\sum_{\ell=2}^{5} n_{k \ell^{\Delta a_{\ell}}}=\frac{g_{k}}{x^{*}+\sum_{\ell=2}^{5} g_{l} \Delta a_{\ell}}-R_{0000}^{k}-Q_{M k}^{\prime}, k=2,3,4,5 .
$$

To use Eq. (18), we first calculate the inverse of $h_{k} \ell$ because it is used many times. We then set $\mathrm{Q}^{\prime}{ }_{\mathrm{Mx}}=0$ and $\Delta \mathrm{a}_{\ell}=0$ on the right-hand side of Eq. (18) and solve for $\Delta \mathrm{a}_{\ell}$ on the left-hand side. With $Q_{M k}^{\prime}$ still zero, we enter the results into the right-hand side and again solve for $\Delta a_{\ell}$ on the left-hand side. The iteration continues until $\Delta \mathrm{a}_{\ell}$ converges. We then enter the $Q_{M r}^{\prime}$ term with $M=2$ and continue the iteration until $\Delta a_{\ell}$ converges. We then enter $M=3$, etc., until successive values of $M$ give the same results.

We successfully applied the maximum likelihood method, using the above iteration scheme, in a Monte Carlo run of $5 \times 10^{5}$ events. In fact, we needed to go up only to $M=2$ to extract the correct Michel parameters (the $\mathrm{V}$-A values in this case) to within one or two standard deviations. Table I shows the result 3 from the Monte Carlo run.

The last column in Table I, taken from a separate Monte Carlo run of $3 \times 10^{6}$ events, shows the type of error that results when the iteration is stopped at $M=2$ instead of $M=3$. Of course, we loaded the deck slightly by generating Monte Carlo data with the V-A values. As the statistics increase, we would expect $\Delta a_{\ell}$ to decrease and, correspondingly, the cutoff to decrease or stay the same; still, the results confirm that $M$ need not be large.

Table II shows the number of $\mathrm{R}$ factors required for a given cutoff.

Before beginning the error analysis, we present a slightly different notation for the Michel parameters (Table III). The notation $b_{k}$. which symbolizes the hare Michel parameters, is related to $a_{k}$ by $a_{k}=b_{k} c_{k}$.

To determine the error matrix, we use $\mathrm{E}_{\mathrm{k} \ell}$ as the error matrix and $\mathrm{F}_{\mathrm{k}}^{-1} \ell$ as an element of the inverse of $\mathrm{E}$. The maximum likelihood method gives us a prescription for finding $\mathrm{E}^{-1}$.

$$
E_{k \ell}^{-1}=-\frac{\partial^{2} \ln G}{\partial b_{k} \partial b_{\ell}}, \quad k, l=2,3,4,5 .
$$

\section{TABLE I}

\begin{tabular}{|c|c|c|c|}
\hline Michel Parameter & Monte Carlo & V-A & $\mathbf{M}=\mathbf{3}$ Correction \\
\hline$p$ & $0.757 \pm 0.004$ & $3 / 4$ & 0.000008 \\
\hline$\eta$ & $0.22 \pm 0.11$ & 0 & 0.0003 \\
\hline$\xi$ & $1.005 \pm 0.018$ & 1 & 0.000009 \\
\hline$\delta$ & $0.757 \pm 0.012$ & $3 / 4$ & 0.000008 \\
\hline
\end{tabular}

\section{MONTE CARLO RESULTS}




\begin{tabular}{cc} 
M & R Factors \\
\cline { 2 - 2 } & \\
1 & 20 \\
2 & 60 \\
3 & 140 \\
4 & 280 \\
5 & 504
\end{tabular}

$\begin{array}{lll}1 & 1 & 1 \\ 2 & p & 1 \\ 3 & \eta & 1 \\ 4 & \xi & \mathrm{p} \\ 5 & \delta & \mathrm{p} \xi\end{array}$

Note that $\mathrm{G}$ is defined in Eq. (8). Working out the derivatives, we obtain

$$
E_{k \ell}^{-1}=N c_{k} c_{l}\left[\frac{1}{N} \sum_{i=1}^{N} \frac{F_{k}\left(x_{j}\right) F_{\ell}\left(x_{j}\right) \mu_{i}^{n_{k}+n_{l}}}{P_{0}\left(x_{f}, \mu_{j}\right)^{2}}-\frac{g_{k} g_{l}}{x^{2}}\right] .
$$

In principle, we could use the Michel parameters that were extracted by using the maximum likelihood method instead of the $\mathrm{V}$-A values to determine the error matrix. However, because there is so little difference between the two, we chose to use the V-A values.

The error matrix could be determined by a Monte Carlo calculation, which makes the results depend on $N$. However, it is more efficient to change Eq. (20) into an analytical expression, which, except for the $\mathrm{N}$ factor in front, is independont of $\mathrm{N}$. This change is possible because $N^{-1} \sum_{1=1}^{N}$ is an average. More precisely, if we let $f(x, \mu)$ stand for some reasonably well-behaved function of $\mathrm{x}$ and $\mu$, then

$$
\frac{1}{N} \sum_{i=1}^{N} f\left(x_{i}, \mu_{i}\right) \longrightarrow \int_{0}^{l} d x \int_{-1}^{l} d \mu P(x, \mu) f(x, \mu),
$$

where $\mathrm{N}$ is large. By making the above substitution, we get

$$
E_{k \ell}^{-1}=N c_{k} c_{\ell}\left[\frac{1}{x} \int_{0}^{l} F_{k}(x) F_{\ell}(x) d x \int_{-1}^{1} \frac{A(x, \mu) \mu^{n_{k}+n_{l}}}{P_{0}(x, \mu)} d \mu-\frac{g_{k} g_{\ell}}{x^{2}}\right] .
$$

Note the complete isolation of $\mathrm{N}$. Now we can write

$$
E_{k \ell}=\frac{1}{N} e_{k \ell},
$$

where $e_{\mathrm{k} \ell}$, a sort of normalized error matrix, is the inverse of Eq. (21) without the $\mathrm{N}$.

Based upon the configuration of our apparatus, including cuts on TPC tracks, we integrated Eq. (21) numerically and found $e_{k \ell}$. The results are as follows. 


$$
e_{k \ell}=\left\{\begin{array}{llll}
5.186 \times 10^{0} & 1.037 \times 10^{2} & 3.781 \times 10^{0} & 2.879 \times 10^{0} \\
& 3.751 \times 10^{3} & 8.546 \times 10^{1} & 7.552 \times 10^{1} \\
& & 9.709 \times 10^{1} & 5.340 \times 10^{1} \\
& & & 4.065 \times 10^{1}
\end{array}\right\}
$$

The correlation matrix is

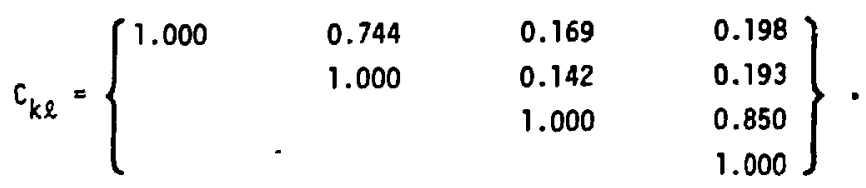

Here we evaluate the statistical errors of the Michel parameters, $\sqrt{e_{k k} / N}$, for $N=10^{\circ}$ events. Table IV shows the current parameter limits given in Ref. 2 . The accuracy of every parameter is improved by at least an order of magnitude. For $\eta$, we see a vast improvement. Note, however, that our results refer to the statistical errors only, whereas the numbers quoted by Sachs and Sirlin ${ }^{2}$ include all sources of error from several experiments.

With such a reduction in the errors, we must determine the limits to be set on the coupling constants. The following coupling constants are written in the same manner as shown in Ret. 2.

$$
x_{i}=\left(\frac{\left|g_{j}\right|^{2}+\left|g_{j}^{\prime}\right|^{2}}{\left|g_{V}\right|^{2}+\left|g_{V}^{\prime}\right|^{2}}\right)^{1 / 2}, i=S, P, T, A,
$$

and

$$
\phi_{V A}=\cos ^{-1}\left(\frac{\operatorname{Re}\left(g_{A}^{*} g_{V}^{\prime}+g_{A}^{\prime} g_{V}^{*}\right)}{\left(\left|g_{V}\right|^{2}+\left|g_{V}^{\prime}\right|^{2}\right)^{1 / 2}\left(\left|g_{A}\right|^{2}+\left|g_{A}^{\prime}\right|^{2}\right)^{1 / 2}}\right) .
$$

Using Derenzo's method ${ }^{8}$ to determine the parameter limits, we present in Table $\mathrm{V} \mathrm{h}_{\text {:i }}$ results

\begin{tabular}{|c|c|c|}
\hline Parameter & $\sqrt{e_{k k} / N}$ & Current Accuracy $^{2}$ \\
\hline$\rho$ & 0.00025 & 0.003 \\
\hline$\eta$ & $0 . \cong j 61$ & 0.21 \\
\hline$\xi$ & 0.00099 & 0.014 \\
\hline$u$ & 0.00064 & 0.009 \\
\hline
\end{tabular}
and the limits we can set from our experiment.

TABLE IV

\section{MICHEL PARAMETER ERRORS}


TABLF $\mathbf{V}$

\section{LIMITS ON THE COUPLING CONSTANTS}

\begin{tabular}{lrr} 
COUPLING CONSTANT & OUR LIMITS & DERENZO'S LIMITS \\
\cline { 1 - 1 } Axial vector & & \\
Tensor & $0.988 \leq \mathrm{x}_{\mathrm{A}} \leq 1.052$ & $0.76 \leq \mathrm{x}_{\mathrm{A}} \leq 1.20$ \\
Scaler & $\mathrm{x}_{\mathrm{T}} \leq 0.027$ & $\mathrm{x}_{\mathrm{T}} \leq 0.28$ \\
Pseudoscaler & $\mathrm{x}_{\mathrm{g}} \leq 0.048$ & $\mathrm{x}_{\mathrm{S}} \leq 0.33$ \\
Vector axial & $\mathrm{x}_{\mathrm{P}} \leq 0.048$ & $\mathrm{x}_{\mathrm{P}} \leq 0.33$ \\
$\quad$ vector phase & $0^{\circ} \leq \Phi_{\mathrm{va}} \leq 2.6^{\circ}$ & $0 \leq \Phi_{\mathrm{vn}} \leq 15^{\circ}$
\end{tabular}

If we limit the other couplings to zero, we get more symmetrical limits, $0.988 \leq \mathrm{x}_{\mathrm{A}} \leq 1.012$, on the axial vector to vector coupling.

The maximum likelihood method seems to be a more efficient way of extracting the Michel parameters from the many data we expect. Just as important, even before the beam is turned on, the method gives us a definitive statement on the expected statistical errors for our apparatus. The results look encouraging, If we can limit the systematics to the same level, a data rur of $10^{\circ}$ events will verify (or possibly even disprove) the V-A values with a sensitivity that is an order of magnitude better than shown in previous experiments.

\section{REFERENCES}

1. M. Bardon, P. Norton, J. Peoples, A. M. Sachs, and J. Lee-Franzini, "Measurement of the Momentum Spectrum of Positrons from Muon Decay," Phys. Rev. Lett. 14, 449 (1965); J. Peoples, "Positron Snectrum from Muor Decay," Ph.D. thesis, Nevis report 147, Columbia University reprint (1966); B. A. Sherwood, "Measurement of the Muon-Decay spectrum with a Wire Spark-Chamber Spectrometer," Phys. Rev. 156, 1475 (1967); David Fryberger, "Measurement of the Muon-Decay Spectrum with a Wire Spark-Chamber Spectrometer," Phys. Rev. 160, 1379 (1968).

2. A. M. Sachs and A. Sirlin, Muon Physics, V. W. Hughes and C. S. Wu, Eds. (Academic Press, New York, 1977), Vol. II, Sec. 2.

3. S. E. Derenzo, "Measurement of the Low-Energy End of the $\mu^{+}$Decay Spectrum," Phys. Rev. 181, 1854 (1969). 